\title{
Commentary on "Delayed, two-staged autologous breast reconstruction: an approach to improving delayed reconstructive outcomes" by AA Patel, L Cai, S Moshrefi, IC Sando, GK Lee \& RS Nazerali
}

\author{
Angelos Mantelakis $^{1}$ (D) $\cdot$ Dianna Kazzazi $^{1} \cdot$ Oliver Bloom $^{1} \cdot$ Michael Gallagher $^{1} \cdot$ Ross Weale $^{1} \cdot$ Jenny Geh $^{1}$
}

Received: 5 January 2021 / Accepted: 26 January 2021 / Published online: 9 February 2021

(C) The Author(s), under exclusive licence to Springer-Verlag GmbH, DE part of Springer Nature 2021

Dear Sir,

We read with great interest the article entitled 'Delayed, two-staged autologous breast reconstruction: an approach to improving delayed reconstructive outcomes' [1]. The authors describe a distinct two-stage breast reconstruction for patients who elect to undergo autologous reconstruction, with tissue expander placement after the initial mastectomy. It is an appealing technique which mitigates the drawbacks of the commonly utilised muscle-sparing transverse rectus abdominal (MS-TRAM) or deep inferior epigastric perforator (DIEP) flaps, including quality and texture mismatch, hair-bearing tissue transfer and scarring. We commend the authors on their outcomes presented in this study, but have a few reservations on the patient selection, surgical technique and outcome measures:

1. Delayed tissue expansion after radiotherapy is frequently encountered in clinical practice in patients who opt for implant reconstruction [2]. The authors suggest that this is the first study to have utilised pre-pectoral tissue expander (PP-TE) placement followed by autologous breast reconstruction, which is not accurate. The exact same technique has been described by Lee et al. in 2019, where the authors utilised PP-TE following mastectomy, which allows the mitigation of the common cosmetic drawbacks of DIEP reconstruction [3].

Angelos Mantelakis

angelos.mantelakis@nhs.net

1 Department of Plastic Surgery, St Thomas' Hospital, Guy's and St Thomas' NHS Foundation Trust, 3rd Floor Lambeth Wing, Westminster Bridge Road, London SE1 7EH, UK
2. The authors performed a retrospective review of all patients who underwent the described procedure over the past 12 years at Stanford University Hospitals. Following this, five patients (eight breasts) were included in this review. Further information on the rationale behind this modest number of included patients would be of interest. Was this because of strict patient selection criteria or a result of patients subsequently choosing to keep the implant-based reconstruction, without proceeding to the autologous transfer?

3. The patients included in the review were non-smokers, non-obese and with minimal or no co-morbidities, thereby increasing the probability of a successful reconstructive outcome [4]. It is unclear from the current manuscript on whether these factors were used in patient selection, and whether the presence of risk factors should deter the utilisation of this technique.

4. The sub-type of breast cancer affects reconstructive options in terms of lymph-node dissection and need for radiation or hormonal therapy. This can also affect the patient and medical team's choice of reconstructive management [4]. This information would help to identify which patient cohort may be most suitable for this technique.

5. It is also not clear which type of autologous surgical reconstruction was used in this study. Which reconstructions were performed (for example, DIEP versus latissimus dorsi within the skin envelope) and what is the authors' preference when performing the described technique?

6. The COVID-19 pandemic has generated a significant elective case backlog due to cessation of all non-emergency cases, which still continues to date [5]. The described method of breast reconstruction is a two-stage operation, with the definitive reconstruction being performed 6 months after the initial tissue expander placement. In the current public health climate, this procedure may result in significant delay to 
definitive reconstruction and this should be taken into account in offering this operation to patients. Also, does this not really equate to a three-stage procedure?

7. The outcome measures include rates of major and minor complications, and a comparison of aesthetic outcomes with matched historical controls. The driver behind the incorporation of reconstruction in the surgical management of breast cancer is the positive impact on patient's quality of life which may be quantified with patient-reported outcome measures (PROMs). Given that the described technique requires the patient to undertake an additional surgical operation, with an additional 6-month delay to definitive reconstruction, PROMs or quality of life data, would be an invaluable addition to this manuscript.

Delayed, two/three-staged autologous breast reconstruction represents an acceptable treatment option in patients who underwent an initial mastectomy with subsequent tissue expander placement, but later opt for autologous breast reconstruction. However, given the requirement for an additional operative procedures and significant delay to definitive reconstruction, this option should be offered based on clear patient inclusion criteria, at the right time and its acceptability needs to be clarified with the target patient population.

\section{Declarations}

Ethical approval Not Applicable
Informed consent Not Applicable

Conflict of interest The Angelos Mantelakis, Dianna Kazzazi, Oliver Bloom, Michael Gallagher, Ross Weale and Jenny Geh declare no conflict of interest.

\section{References}

1. Patel AA, Cai L, Moshrefi S, Sando IC, Lee GK, Nazerali RS (2020) Delayed, two-staged autologous breast reconstruction: an approach to improving delayed reconstructive outcomes. Eur J Plast Surg 43(5):563-568

2. Seth AK, Silver HR, Hirsch EM, Kim JY, Fine NA (2015) Comparison of delayed and immediate tissue expander breast reconstruction in the setting of postmastectomy radiation therapy. Ann Plast Surg 75(5):503-507

3. Lee KR, Clavin N (2019) Bridging autologous reconstruction with pre-pectoral tissue expanders. Gland Surg 8(1):90-94

4. Szloch J, Marczyk E, Kołodziej-Rzepa M, Komorowski AL (2016) Impact of different type of cancer treatment on the effectiveness of breast reconstruction. Gland Surg 5(4):444-449

5. Macdonald N, Clements C, Sobti A, Rossiter D, Unnithan A, Bosanquet N (2020) Tackling the elective case backlog generated by Covid-19: the scale of the problem and solutions. J Public Health 42(4):712-716

Publisher's note Springer Nature remains neutral with regard to jurisdictional claims in published maps and institutional affiliations. 